\title{
Transcranial Random Noise Stimulation of Visual Cortex: Stochastic Resonance Enhances Central Mechanisms of Perception
}

\author{
Onno van der Groen ${ }^{1,3}$ and Nicole Wenderoth ${ }^{1,2,3}$ \\ ${ }^{1}$ Neural Control of Movement Laboratory, Department of Health Sciences and Technology, ETH Zurich, 8057 Zurich, Switzerland, ${ }^{2}$ Movement Control and \\ Neuroplasticity Research Group, Department of Kinesiology, Biomedical Sciences Group, KU Leuven, 3000 Leuven, Belgium, and ${ }^{3}$ Neuroscience Centre \\ Zurich, 8057 Zurich, Switzerland
}

Random noise enhances the detectability of weak signals in nonlinear systems, a phenomenon known as stochastic resonance (SR). Though counterintuitive at first, SR has been demonstrated in a variety of naturally occurring processes, including human perception, where it has been shown that adding noise directly to weak visual, tactile, or auditory stimuli enhances detection performance. These results indicate that random noise can push subthreshold receptor potentials across the transfer threshold, causing action potentials in an otherwise silent afference. Despite the wealth of evidence demonstrating SR for noise added to a stimulus, relatively few studies have explored whether or not noise added directly to cortical networks enhances sensory detection. Here we administered transcranial random noise stimulation (tRNS; $100-640 \mathrm{~Hz}$ zero-mean Gaussian white noise) to the occipital region of human participants. For increasing tRNS intensities (ranging from 0 to $1.5 \mathrm{~mA}$ ), the detection accuracy of a visual stimuli changed according to an inverted-Ushaped function, typical of the SR phenomenon. When the optimal level of noise was added to visual cortex, detection performance improved significantly relative to a zero noise condition $(9.7 \pm 4.6 \%)$ and to a similar extent as optimal noise added to the visual stimuli $(11.2 \pm 4.7 \%)$. Our results demonstrate that adding noise to cortical networks can improve human behavior and that tRNS is an appropriate tool to exploit this mechanism.

Key words: perception; stochastic resonance; tRNS

\section{Significance Statement}

Our findings suggest that neural processing at the network level exhibits nonlinear system properties that are sensitive to the stochastic resonance phenomenon and highlight the usefulness of tRNS as a tool to modulate human behavior. Since tRNS can be applied to all cortical areas, exploiting the SR phenomenon is not restricted to the perceptual domain, but can be used for other functions that depend on nonlinear neural dynamics (e.g., decision making, task switching, response inhibition, and many other processes). This will open new avenues for using tRNS to investigate brain function and enhance the behavior of healthy individuals or patients.

\section{Introduction}

Noise is as an intrinsic property of all biological systems and is typically viewed as being detrimental for neural computations and the associated behavior. However, when an appropriate level

\footnotetext{
Received Dec. 18, 2015; revised March 22, 2016; accepted March 26, 2016.

Author contributions: $0 . v$. ..G. and N.W. designed research; $0 . v$. ..G. analyzed data; $0 . v$.d.G. and N.W. wrote the paper.

This work was supported by Swiss National Science Foundation Grant 320030_149561 and Research Foundation-Flanders Grant G040112N. We thank the SNSF and FWO for their financial support and Daniel Woolley for his help and comments throughout this project.

The authors declare no competing financial interests.

Correspondence should be addressed to Onno van der Groen, Neural Control of Movement Laboratory, Department of Health Sciences and Technology, ETH Zurich, Winterthurerstrasse 190, Building Y36/Y17, Floor M, 8057 Zurich, Switzerland. E-mail: vandergroen@gmail.com.

DOI:10.1523/JNEUROSCI.4519-15.2016

Copyright $\odot 2016$ the authors $\quad 0270-6474 / 16 / 365289-10 \$ 15.00 / 0$
}

of random noise is added to a nonlinear system, it can enhance weak signals, an effect known as stochastic resonance (SR). For example, adding an optimal level of noise to weak visual (Riani and Simonotto, 1994; Simonotto et al., 1997; Ward et al., 2001), tactile (Collins et al., 1995; Collins et al., 1996), or auditory stimuli (Zeng et al., 2000) has been shown to significantly enhance detection performance. SR is characterized by a larger effect for subthreshold stimuli than for suprathreshold stimuli (Gingl et al., 1995), and the signal enhancing effect depends on the intensity of the added noise according to an inverted-U-shaped curve. The inverted-U-shaped curve is a hallmark of the SR phenomenon and indicates that the level of added noise needs to be optimal to maximally enhance performance.

Most previous studies added noise to the stimulus (single receptor design) so that signal and noise could already interact in 
the peripheral nervous system, which contains nonlinear neural elements like sensory receptors. However, SR effects are certainly not limited to the peripheral nervous system. For example, it has been shown that visual detection performance can be enhanced by adding visual noise to one eye and a subthreshold visual signal to the other (Kitajo et al., 2003). This suggests that neural processing is sensitive to the SR phenomenon. However, it is not clear whether it emerges at the cortical or subcortical level because inputs from both eyes interact in the lateral geniculate nucleus of the thalamus (Wunderlich et al., 2005; Tong et al., 2006) and primary visual cortex.

With the previous development of transcranial random noise stimulation (tRNS) (Terney et al., 2008; Chaieb et al., 2015), it is now possible to add noise to the cortex with well-controlled frequency, duration, and intensity characteristics. Although the physiological mechanism of tRNS is not completely understood, it has been demonstrated that a short period of repetitive extracellular high-frequency stimulation in rat neurons resulted in an inward sodium current, which caused a weak depolarization of the cell membrane (Bromm, 1968; Schoen and Fromherz, 2008). Previous work in humans has demonstrated physiological and behavioral aftereffects following prolonged tRNS stimulation (Terney et al., 2008; Fertonani et al., 2011). For example, $10 \mathrm{~min}$ of tRNS increased the cortical excitability for up to an hour after stimulation (Terney et al., 2008), and $\sim 22$ min of tRNS during a perceptual learning task improved behavioral performance (Fertonani et al., 2011). In these studies, it has been suggested that SR plays a role in tRNS, but this has not been tested directly. Importantly, if tRNS can influence behavior via an SR mechanism, the effect should be observable online and dependent on the noise and stimulus intensity. In the present study, we tested whether visual detection performance can be enhanced in an SR-like manner when different noise intensities are applied to the visual cortex while performing a visual detection task of varying difficulty.

First, we replicated previous studies by testing if adding noise to the visual stimulus directly (visual-noise experiment) enhances detection performance in a manner consistent with the SR phenomenon. We then tested whether adding noise to the visual cortex with tRNS (tRNS-noise experiment) enhances visual detection performance. Finally, we applied tRNS to the forehead (i.e., over frontal lobe) to determine whether it has unspecific effects on detection performance (tRNS-control experiment). We predicted that the addition of noise would improve the detection of subthreshold stimuli more than suprathreshold stimuli in both the visual-noise and tRNS-noise experiments, but not in the tRNS-control experiment. For both visual-noise and tRNSnoise, we expected to find an inverted-U-shaped relationship between noise intensity and detection performance, indicating that performance is only enhanced when an optimal level of noise is added.

\section{Materials and Methods}

Power analysis and subjects

An a priori power analysis ( $\mathrm{G}^{\star}$ Power version 3.1.3; Faul et al., 2007) indicated that a sample size of seven subjects per group would be sufficient to detect a significant effect on detection performance in the visualnoise experiment with a power of 0.80 for an $\alpha$ level of 0.05 . These values are based on previous work investigating the effect of noise on the detection of a subthreshold visual signal (Sasaki et al., 2008). In that study, noise added directly to the visual stimulus had a large effect on signal detection (Cohen's $d=1.71, n=19$ ). In our experiments, we included more subjects to ensure sufficient power in the tRNS experiments, since it was not possible to estimate the effect of tRNS on a visual detection task.
A total of 52 healthy right-handed subjects took part in at least one of three separate experiments (26 female; mean age, 24; age range, 18-30). All subjects had normal or corrected-to-normal vision and met the inclusion criteria for tRNS. Subjects were allowed to participate in different experiments, but we ensured that experimental sessions were separated by at least $24 \mathrm{~h}$ and that the order of participating in different experiments was counterbalanced (below detailed descriptions of subject characteristics are reported for each experiment separately).

The study was approved by the Human Research Ethics Committee, ETH Zürich (EK 2013-N-55) and the Kantonale Ethik Komission Zürich, Switzerland (KEK-ZH-Nr. 2014-0269). Informed consent was obtained from all participants before the start of the experiment.

\section{General procedure}

Stimulus and apparatus. All experiments took place in a dark and quiet room. Participants were seated $1 \mathrm{~m}$ away from a screen on a comfortable chair. Visual stimuli were generated using Matlab version 2012b and the Psychophysics toolbox (Brainard, 1997; Pelli, 1997; Kleiner et al., 2007). Stimuli were presented on a Sony CPD-G420 color monitor with a calibrated linearized output at a resolution of $1280 \times 1024$ pixels and a refresh rate of $75 \mathrm{~Hz}$. The visual target stimulus was a vertically orientated Gabor patch (a sinusoidal luminance pattern presented within a Gaussian envelope) in a soft circular aperture with a vertical orientation. The target extended a visual angle of $1.4^{\circ}$, with a spatial frequency of 2.9 cycles per $\mathrm{cm}$ and a mean luminance of $52 \mathrm{~cd} / \mathrm{m}^{2}$. Stimuli were presented on a uniform gray background $\left(52 \mathrm{~cd} / \mathrm{m}^{2}\right)$. The Gabor patch was always presented within one of eight locations (indicated by white circles on the screen, $1.5^{\circ}$ visual angle), which were positioned around the fixation cross $\left(0.6^{\circ}\right.$ visual angle) as shown in Figure $1 A$. The stimuli and apparatus were the same for all three experiments.

Two-alternative forced choice visual detection task. In all three experiments participants performed a two-alternative forced choice (2-AFC) visual detection task. Each trial started with a green fixation cross (visual angle $0.6^{\circ}$ ). After a key press there was a beep, and eight circles appeared for $2.04 \mathrm{~s}$ at potential target locations on the screen (first interval). Then a blank screen was shown for $1 \mathrm{~s}$ and the eight circles reappeared for another $2.04 \mathrm{~s}$ (second interval). Subjects were instructed that the visual target stimulus (Gabor patch) was presented at one of the eight potential locations either during the first or second interval. After the second interval ended, the fixation cross turned red, prompting subjects to make a forced-choice judgment to indicate which interval contained the visual target. After participants responded, there was a break of $3 \mathrm{~s}$ before the next trial started. The target was presented the same number of times at each of the eight possible locations to account for the possibility that detection performance may be location dependent (Fig. 1A). Furthermore, all presentation locations were situated on the noncardinal axes since it has been shown that detection performance on these axes does not vary greatly compared to detection performance on the cardinal axes (Cameron et al., 2002). We used a Tobii eye tracker (model tx300; sampling frequency, $300 \mathrm{~Hz}$ ) to control for eye movements and blinks during stimulus presentation in both the thresholding block and the main experiment. When eye movements or blinks were detected, the trial was repeated. In all experiments, participants performed five blocks of 40 trials, with a different noise intensity tested in each block. The detection task was the same in all three experiments, however, in the visual-noise experiment, noise was added to all eight circles on the screen, and in the tRNS-noise and tRNS-control experiments, noise was applied via tRNS.

Determining individual target stimulus intensities. At the start of each experiment, we determined each individual's target contrast detection threshold with a thresholding block. In the thresholding block, the same 2-AFC task was used as in the main experiment without presentation of any noise, but the intensity of the grating was adapted to determine the visual detection threshold of each subject. We determined the stimulus contrast that corresponded to $75 \%$ detection accuracy during the 2-AFC task using a QUEST staircase procedure (thresholding block) (Watson and Pelli, 1983). The thresholding block started by determining a rough estimate of the participant's detection threshold value with a simple updown staircase method. After five reversals of their yes-no responses, we stopped the staircase and calculated an estimate of the individual detec- 
A
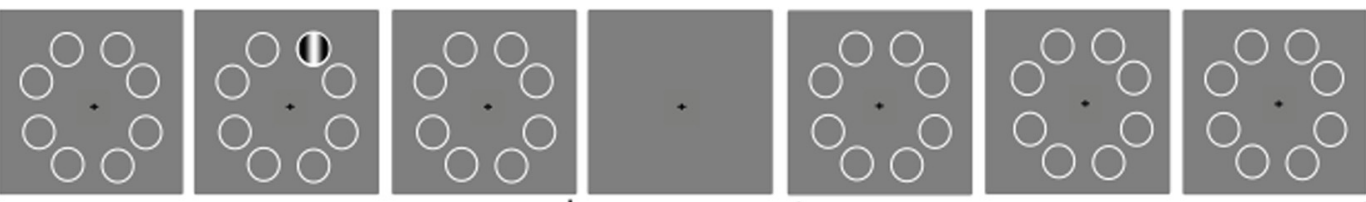

$$
1000 \mathrm{~ms} \quad 40 \mathrm{~ms} \quad 1000 \mathrm{~ms}
$$

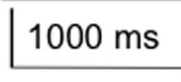

$40 \mathrm{~ms}$

$1000 \mathrm{~ms}$

B tRNS OR Visual noise

\section{Experiment 1 \\ Visual-Noise}
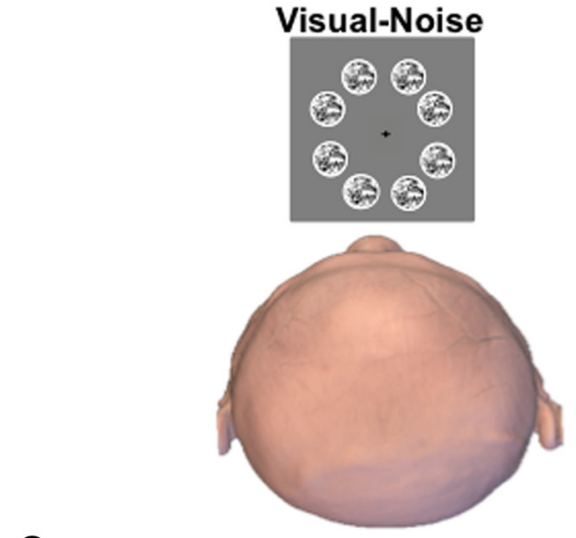

C

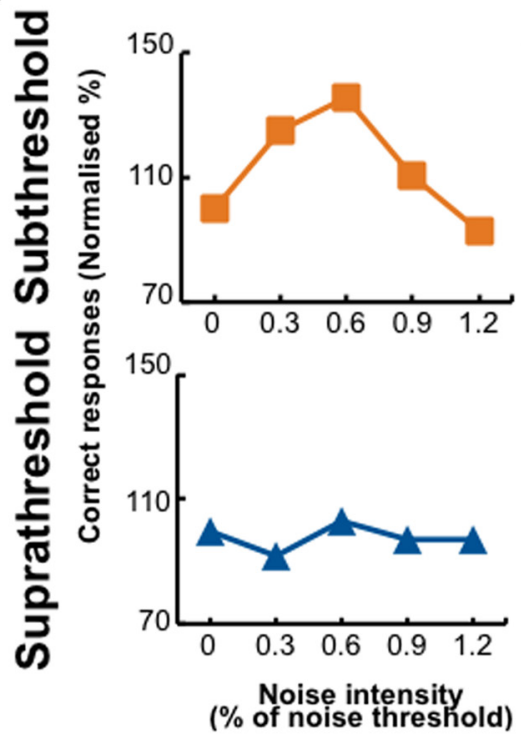

\section{Experiment 2} tRNS-Noise

Second Interval tRNS OR Visual noise

\section{Experiment 3 tRNS-Control}
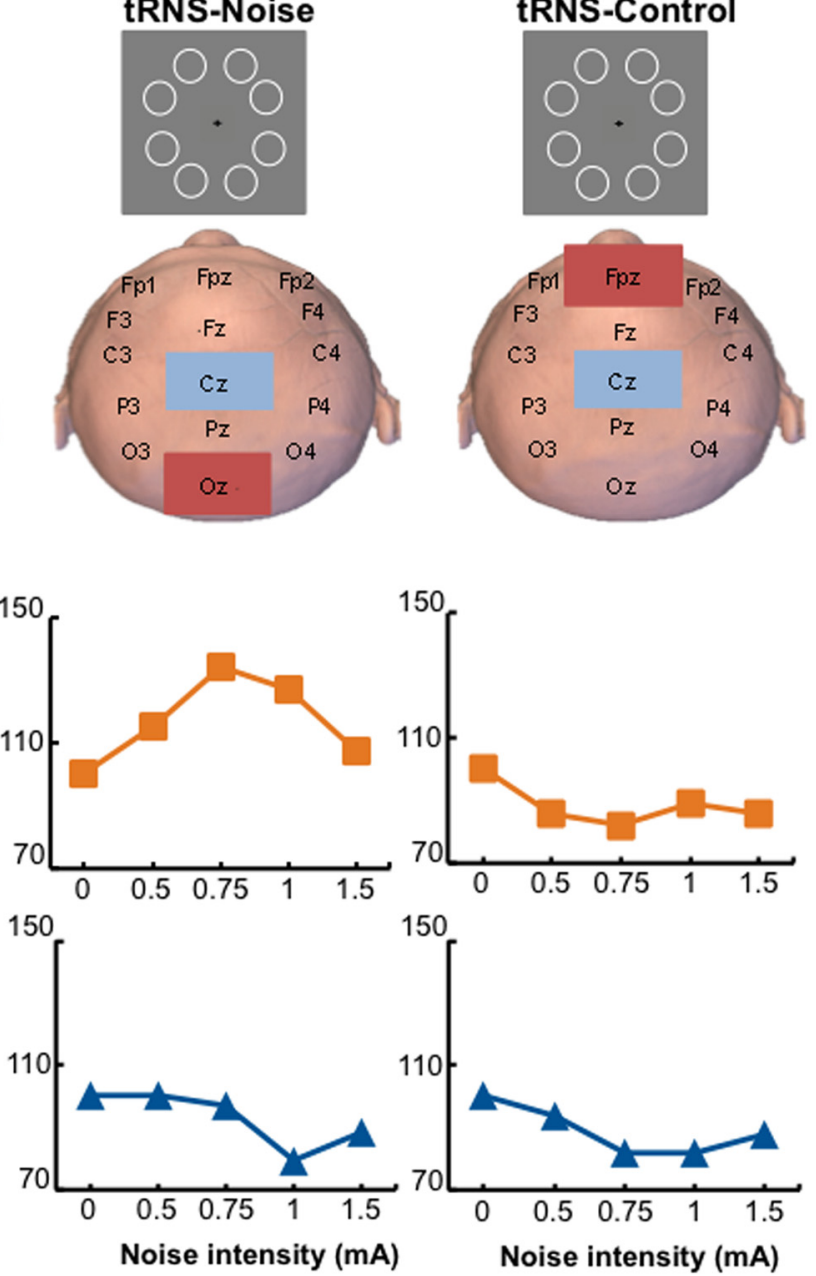

Figure 1. Experimental setup. $\boldsymbol{A}$, The 2-AFC visual detection task. Subjects fixated on the center of the screen and a visual stimulus was randomly presented either in the first (shown here) or second interval. After the second interval, participants had to indicate which interval contained the stimulus. In the main experiment, the stimulus contrast was fixed to yield either $60 \%$ detection accuracy (subthreshold group) or $80 \%$ detection accuracy (suprathreshold group). $\boldsymbol{B}$, Representation of the three different experiments. Note that in the tRNS-noise and tRNS-control experiments, no noise was presented on the screen. C, Representative data of individual participants. The participants in the visual-noise (left) and tRNS-noise (middle) experiments show a peak in their detection performance when noise was added to a subthreshold stimulus (orange line), but not to a suprathreshold stimulus (blue line).

tion threshold, which we used as an input for the QUEST procedure. Then two QUEST staircases were randomly interleaved, one that started at 1.2 times the estimated threshold, and one at 0.8 times the estimated threshold (40 trials per QUEST). The stimulus contrast was adjusted to yield either 60\% detection accuracy (subthreshold group) or $80 \%$ detection accuracy (suprathreshold group) and was fixed at this value in the main experiment. The procedure to determine individual target stimuli intensities was conducted at the start of every experiment.

Experiment 1: adding visual noise directly to the visual stimulus (visual-noise)

In this experiment, we tested the hypothesis that adding an optimal level of noise to a subthreshold visual target stimulus can improve visual con- trast sensitivity. Participants ( $n=31 ; 14$ female; mean age, 24; age range, 19-30) were randomly assigned to one of two groups and performed a 2-AFC visual detection task (Fig. 1A). One group detected the visual target stimulus presented at a subthreshold contrast level (corresponding to an average detection accuracy of $60 \%$ ), and the other group detected the visual target stimulus presented at a suprathreshold contrast level (corresponding to an average detection accuracy of 80\%).

Visual noise was added together with the visual stimulus on the screen (visual-noise experiment; Fig. 2), and the noise intensity varied among 0 , $0.3,0.6,0.9$, and 1.2 times the individual noise threshold. In this experiment, participants completed an additional block of thresholding in which a QUEST staircase was used to determine a 75\% noise detection threshold. 


\section{Experiment 1: visual stimulus with different noise levels}

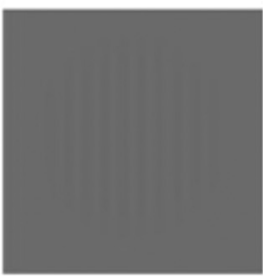

zero-noise

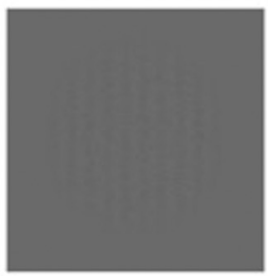

$0.3 \times$ noise threshold

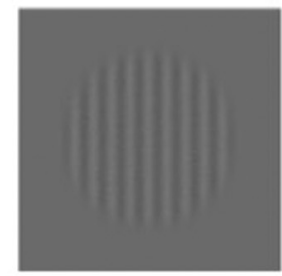

$0.6 \times$ noise threshold

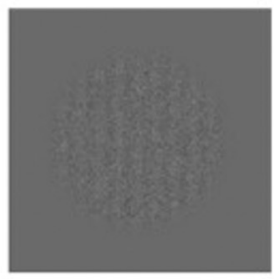

$0.9 \times$ noise threshold

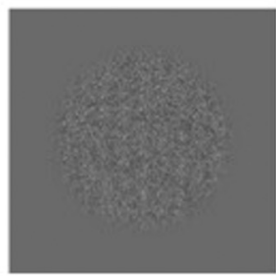

$1.2 \times$ noise threshold

Figure 2. Example of a visual target stimulus with five different noise levels. In the visual-noise experiment, different levels of noise (multiples of individual noise detection thresholds) were added to the target stimulus.

The procedure was the same as for determining individual target stimulus intensities; however, the target was replaced with random Gaussian white noise, which was presented for one complete interval duration (2.04 s) at all eight locations.

\section{Experiment 2: adding electrical noise via tRNS to the visual} cortex (tRNS-noise)

In this experiment, we tested the hypothesis that applying tRNS to the occipital cortex at an optimal intensity can improve the visual contrast sensitivity of a subthreshold visual stimulus. Participants $(n=38 ; 21$ female; mean age, 25; age range, 19-30) were randomly assigned to one of two groups (subthreshold or suprathreshold contrast levels) and performed a 2-AFC visual detection task (Fig. 1A). The target stimulus intensity and visual detection task used were the same as for the visualnoise experiment, except that noise was applied via tRNS to the occipital cortex during visual stimulus presentation (tRNS-noise).

In this experiment, randomly alternating current (frequencies between 100 and $640 \mathrm{~Hz}$ ) of $0,0.5,0.75,1$, or $1.5 \mathrm{~mA}$ intensity (amplitude) with a 0 $\mathrm{mA}$ offset was applied during both intervals of the 2-AFC task for $2.04 \mathrm{~s}$ each. No noise thresholding block was conducted because it is not possible to determine individual noise levels for tRNS noise. Noise was injected centrally with tRNS applied to the visual cortex. Electrode placement was determined using the 10-20 system. The stimulation electrode was placed over the occipital region (Oz in the 10-20 EEG system) and the reference over the vertex (Cz in the 10-20 EEG system; Fig. 1B). This setup is suitable for stimulation of the visual cortex (Neuling et al., 2012). Electroconductive gel was applied to the contact side of the electrode $(5 \times 7 \mathrm{~cm})$ to reduce skin impedance. Electrodes were held in place with a bandage. Stimulation was delivered by a battery-driven electrical stimulator (version DC-Stimulator PLUS, neuroConn). The maximum current density in this study was $42.85 \mu \mathrm{A} / \mathrm{cm}^{2}$, which is within recently updated safety limits (Fertonani et al., 2015). The tRNS waveform was created in Matlab and sent to the DC stimulator via a data acquisition card (National Instruments USB-6343 X series). Modeling was used to estimate the electrical field strength in the visual cortex (Spheres 2.0; Truong et al., 2014). We did this for every tRNS intensity and for specific frequencies between 100 and $500 \mathrm{~Hz}$ (50 Hz steps; Table 1).

\section{Experiment 3: adding electrical noise via $t R N S$ to a control} region ( $t R N S$ - control)

This experiment served as a control to test for unspecific effects of tRNS on visual/detection performance. We tested whether tRNS applied to the forehead can improve the visual contrast sensitivity of a subthreshold visual stimulus. In this experiment, participants ( $n=20$; 9 female; mean age, 25; age range, 21-30) were randomly assigned to one of the two groups and performed the 2-AFC visual detection task (Fig. 1). This experiment followed the same procedure as the tRNS-noise experiment, with the only difference being that the stimulation electrode was placed over the forehead and the reference electrode over the vertex (Cz in the 10-20 EEG system). The noise was again applied in both intervals for $2.04 \mathrm{~s}$.

\section{Data analyses}

The same statistical procedures were applied to all three experiments. The $\alpha$ level was set to 0.05 for all tests.
Table 1. Modeled electric field strength for different transcranial electrical current stimulation intensities and frequencies

\begin{tabular}{lllll}
\hline & \multicolumn{4}{l}{ Intensity $(\mathrm{mA})$} \\
\cline { 2 - 5 } Frequency $(\mathrm{Hz})$ & $0.5 \mathrm{~mA}(\mathrm{~V} / \mathrm{m})$ & $0.75 \mathrm{~mA}(\mathrm{~V} / \mathrm{m})$ & $1 \mathrm{~mA}(\mathrm{~V} / \mathrm{m})$ & $1.5 \mathrm{~mA}(\mathrm{~V} / \mathrm{m})$ \\
\hline $100 \mathrm{~Hz}$ & 0.7 & 1.6 & 2.2 & 3.2 \\
$150 \mathrm{~Hz}$ & 0.8 & 1.5 & 2 & 3.1 \\
$200 \mathrm{~Hz}$ & 1.1 & 1.5 & 2.2 & 3.1 \\
$250 \mathrm{~Hz}$ & 1 & 1.6 & 2.2 & 3 \\
$300 \mathrm{~Hz}$ & 1 & 1.6 & 2.1 & 3 \\
$350 \mathrm{~Hz}$ & 1 & 1.5 & 2.1 & 3 \\
$400 \mathrm{~Hz}$ & 1 & 1.5 & 2.1 & 3 \\
$450 \mathrm{~Hz}$ & 1 & 1.5 & 2.2 & 3 \\
$500 \mathrm{~Hz}$ & 1 & 1.5 & 2.2 & 3 \\
\hline
\end{tabular}

The modeling shows that that all stimulation frequencies were transmitted to the brain and that the current applied is strong enough to reach the cortex (scaling quasi-linearly with tRNS intensity).

Table 2. Overview of effect sizes and obtained statistical power for the influence of noise on detection performance in the different experiments

\begin{tabular}{llllll}
\hline & \multicolumn{2}{l}{ Effect sizes (Cohen's $d$ ) } & & Obtained power & \\
\cline { 2 - 3 } \cline { 5 - 6 } Experiment & Initial analysis & Control analysis & & Initial analysis & Control analysis \\
\hline Visual-noise & 1.15 & 0.94 & 0.85 & 0.68 \\
tRNS-noise & 0.81 & 0.77 & 0.65 & 0.59 \\
tRNS- control & 0.05 & 0.24 & & 0.05 & 0.08 \\
\hline
\end{tabular}

The effect sizes are based on the maximum improvement data for each experiment. This was done for the initial and control analyses.

To quantify the SR effect, we normalized the percentage correct (\%Corr) detection performance to the zero noise condition (all trials where no noise was applied): \%Corr (normalized) $=\% \operatorname{Corr}(i) / \% \operatorname{Corr}$ (zero noise), with $i$ denoting each of the five tested noise intensities. To test for effects of added noise on detection performance, normalized accuracy data were subjected to a repeated measures ANOVA with the within-subjects factor noise intensity (five levels for each experiment) and the between-subjects factor stimulus intensity (subthreshold vs suprathreshold group). Additionally, preplanned comparisons testing performance for each noise level against the zero noise condition were conducted and corrected for multiple comparisons, resulting in an adjusted $\alpha$ level of 0.0125 . It is well known that there are big individual differences in optimal noise levels (Collins et al., 1996; Martínez et al., 2007). Therefore we determined the optimal noise level for each participant, which was defined as the noise level where detection performance was best. The difference in performance between an individual's optimal noise level (i.e., the peak in each graph in Fig. 1C) and zero noise performance (i.e., the first data point in each graph in Fig. 1C) served as a measure of the effectiveness of optimal noise (maximum improvement). Note that a maximum improvement of 0 indicates that there was no SR effect; i.e., adding noise always led to worse performance. Statistical analyses were performed using SPSS (version 20.0, IBM). Finally, we calculated the effect size of the noise induced performance improvements for each of the three different experiments (Table 2). We also determined 


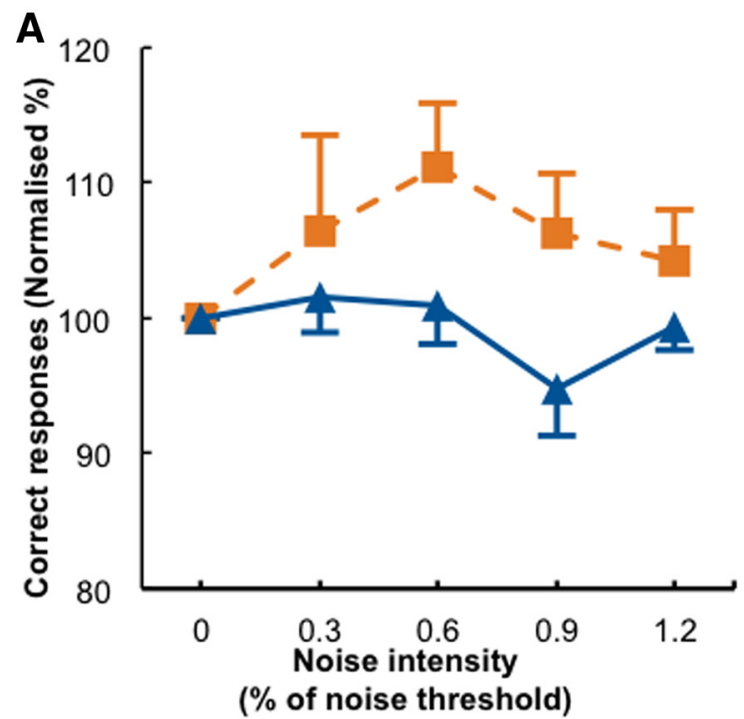

C

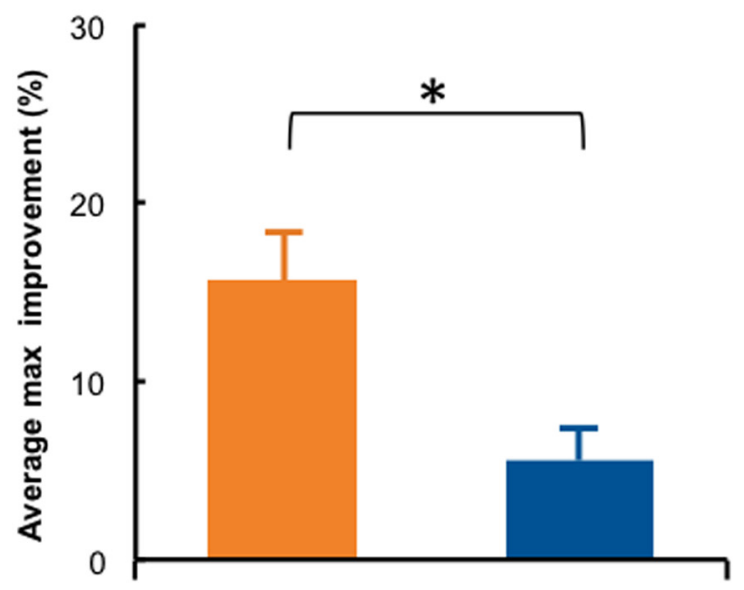

Subthreshold

Suprathreshold

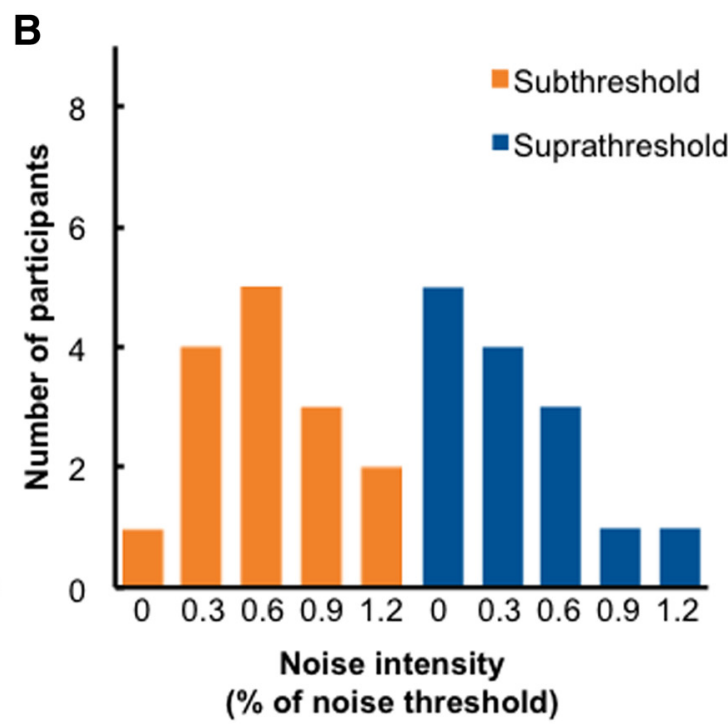

D

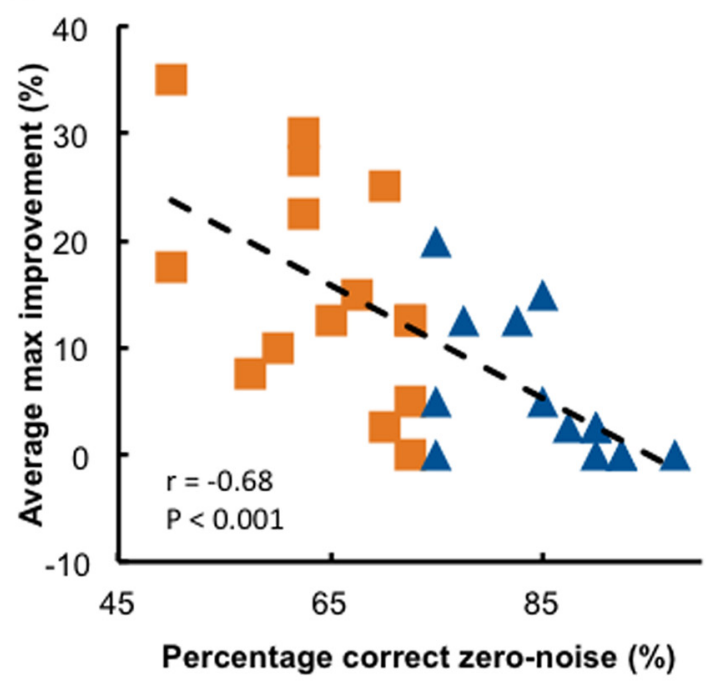

Figure 3. Results when noise is added to the visual stimulus (visual-noise experiment). $\boldsymbol{A}$, Detection accuracy relative to the zero noise condition is shown for each noise level in the subthreshold and suprathreshold stimulus groups. $\boldsymbol{B}$, Distribution of the individual optimal noise levels (defined as the noise intensity that yields maximum accuracy detection). $\boldsymbol{C}$, A comparison between the average maximum improvement (i.e., the percentage difference between maximum accuracy and zero noise accuracy) showed a significant difference between the subthreshold and suprathreshold groups. ${ }^{*} p<0.05$. D, We found a significant correlation between baseline performance and maximal improvement, indicating that participants with low detection accuracy in the zero noise condition benefited most from adding optimal noise. Error bars indicate SEs.

that the actual statistical power of our main experiments was $\sim 0.6$ ( $\mathrm{G}^{\star}$ Power version 3.1.3; Faul et al., 2007).

\section{Additional analysis}

The goal of this additional analysis was to determine the optimal noise level and the associated performance improvement using independent data sets. Therefore, all trials per subject and per noise level were randomly split in half. One-half of the trials (discovery data set) were used to define the optimal noise level (i.e., the noise value which causes a maximum improvement in detection performance), and the other half (test data set) were used to determine the performance improvement observed for the "discovered" optimal noise level when compared to zero noise performance. In this way, determining the optimal noise level and obtaining the associated performance improvement was based on independent data. This procedure was done for all experiments and for all noise levels. The procedure was repeated 1000 times for each participant, yielding an average performance improvement associated with the optimal noise level, and these values were subsequently compared between the subthreshold and suprathreshold groups using the same statistics as described above.

\section{Results}

Visual noise added to the visual stimulus improves detection of subthreshold but not suprathreshold visual stimuli

In the visual-noise experiment, we investigated the influence of adding different levels of visual noise (i.e., presented together with the visual stimulus on the screen) on the detection of either subthreshold or suprathreshold stimuli. Two participants were excluded from the analysis in the subthreshold group because they performed below chance level in the zero noise condition. This left a total number of 29 participants in the analyses (15 in the subthreshold group).

Adding noise to the visual stimulus improved detection accuracy significantly more in the subthreshold stimuli group than in the suprathreshold stimulus group (Fig. 3A; significant group main effect tested with a repeated-measures ANOVA, $F_{(1,27)}=$ $4.59, p=0.041)$. When noise was added to subthreshold stimuli, normalized detection accuracy improved gradually for low to 
Table 3. Overview of the maximum improvement and optimal noise levels for the different experiments and different analysis

\begin{tabular}{|c|c|c|c|c|}
\hline \multirow[b]{2}{*}{ Experiment } & \multicolumn{2}{|l|}{ Initial analysis } & \multicolumn{2}{|l|}{ Control analysis } \\
\hline & $\begin{array}{l}\text { Optimal noise } \\
\text { level mode }\end{array}$ & $\begin{array}{l}\text { Maximum } \\
\text { improvement } \\
(\%, \text { mean } \pm \text { SE) }\end{array}$ & $\begin{array}{l}\text { Optimal noise } \\
\text { level mode }\end{array}$ & $\begin{array}{l}\text { Maximum } \\
\text { improvement } \\
(\%, \text { mean } \pm \text { SE) }\end{array}$ \\
\hline \multicolumn{5}{|l|}{ Subthreshold } \\
\hline Visual-noise & $\begin{array}{r}60 \% \text { of noise } \\
\text { threshold }\end{array}$ & $15.67 \pm 2.69$ & $\begin{array}{r}60 \% \text { of noise } \\
\text { threshold }\end{array}$ & $12.04 \pm 2.83$ \\
\hline tRNS-noise & $1 \mathrm{~mA}$ & $14.38 \pm 2.41$ & $1 \mathrm{~mA}$ & $11.07 \pm 2.27$ \\
\hline tRNS- control & $0 \mathrm{~mA}$ & $5 \pm 2.01$ & $0 \mathrm{~mA}$ & $2.45 \pm 1.65$ \\
\hline \multicolumn{5}{|l|}{ Suprathreshold } \\
\hline Visual-noise & $\begin{array}{l}0 \% \text { of noise } \\
\text { threshold }\end{array}$ & $5.53 \pm 1.78$ & $\begin{array}{r}30 \% \text { of noise } \\
\text { threshold }\end{array}$ & $3.71 \pm 1.55$ \\
\hline tRNS-noise & $0 \mathrm{~mA}$ & $7.34 \pm 1.47$ & $0 \mathrm{~mA}$ & $4.87 \pm 1.18$ \\
\hline tRNS- control & $1.5 \mathrm{~mA}$ & $4.44 \pm 1.54$ & $0 \mathrm{~mA}$ & $1.48 \pm 0.89$ \\
\hline
\end{tabular}

The results of our additional analysis confirmed the results of our optimal noise level analysis even though the performance improvement observed for the optimal noise level is, as expected, smaller in our additional analysis.

medium noise levels. However, when too much noise was added, detection accuracy deteriorated again. Thus, an inverted-Ushaped function was observed when detection accuracy was expressed as a function of the noise intensity, both at the individual (Fig. 1C, orange line, first column, a representative subject) and group levels (Fig. $3 A$, orange line). This pattern of behavior is typical for the SR phenomenon. By contrast, when noise was added to suprathreshold stimuli, detection performance was nearly unchanged (Figs. $1 C$, blue line, first column; $3 A$, blue line). Group differences were most pronounced when noise intensity corresponded to 0.6 times the individual noise detection threshold; however, statistical significance was only reached at an uncorrected level $\left(t_{(14)}=2.48, p=0.026\right.$; preplanned comparisons; corrected $\alpha$ level, 0.0125$)$. This is likely due to high interindividual variability in optimal noise levels, which has also been reported in other SR studies (Collins et al., 1996; Martínez et al., 2007). Therefore, we looked at whether individual participants differed regarding their "optimal" noise level, defined as the noise intensity that led to maximal detection accuracy. Figure $3 B$ shows the distribution of optimal noise levels for the subthreshold (orange) and suprathreshold groups (blue). In the subthreshold group, most, but not all, participants benefited from the 0.6 noise level. In contrast, the participants in the suprathreshold group benefited most from 0 noise, indicating that adding noise to suprathreshold stimuli resulted in poorer detection performance in most cases. We also analyzed the maximum improvement of each participant compared to the zero noise condition (Fig. 3C) and found that the greatest noise-induced improvement in accuracy (percentage change relative to zero noise condition) was significantly larger in the subthreshold group $(15.7 \%)$ then the suprathreshold group $\left(5.5 \%, t_{(27)}=3.10, p=0.005\right)$. Note, however, that the above analysis contains an element of intrinsic circularity because the optimal noise level and the maximal improvement of each individual were based on the same data set. Although this procedure is unlikely to compromise the between-group comparison, it certainly overestimates the size of the stochastic resonance effect in the visual-noise experiment. We therefore performed a control analysis that determined the optimal noise level and the associated detection performance improvement independently. This control analysis confirmed that adding an optimal level of visual noise to subthreshold stimuli improved detection accuracy by $12.04 \%$, which is significantly larger than adding noise to suprathreshold stimuli $\left(12.04\right.$ vs $3.70, t_{(21.981)}=$ $2.563, p=0.018$; for an overview of the maximum improvement and optimal noise levels for all experiments and both analyses, see Table 3). Effect size analyses revealed that adding noise had a large effect on detection performance when the subthreshold stimuli group was compared to the suprathreshold stimuli group (Table 2). Finally, we found a significant correlation between absolute detection accuracy in the zero noise condition and the maximum noise-induced improvement $\left(r_{(27)}=-0.68, p<\right.$ 0.001; Fig. 2D). Thus, participants who had to detect a visual stimulus below their detection threshold benefitted more from added noise than participants that had to detect a visual stimulus at or just above their detection threshold (Fig. 3D). One general concern is that the results of the suprathreshold group might have been confounded by a ceiling effect. However, only one participant in the suprathreshold group reached $100 \%$ accuracy when noise was added to the stimuli, and this participant's maximum improvement (17\%) clearly exceeded the group mean of $5.5 \%$. In summary, we replicated and extended previous findings showing that SR effects are significantly larger for subthreshold than for suprathreshold stimuli using a conventional paradigm that adds visual noise to a visual detection task.

\section{Adding noise centrally to the visual cortex with tRNS improves detection of subthreshold but not suprathreshold stimuli}

The tRNS-noise experiment used the same visual detection task as the visual-noise experiment, but instead of presenting noise visually on the screen, it was applied centrally to the visual cortex using tRNS with intensities of $0,0.5,0.75,1$, and $1.5 \mathrm{~mA}$.

Two participants were excluded from the analysis because one performed below chance level (subthreshold group) and one displayed a ceiling effect (suprathreshold group) in the zero noise condition. This left a total number of 36 participants in the analyses (20 in the subthreshold group).

To test how much current reached the brain, we used a simplified spherical model to simulate the electrical field distribution induced by different frequency components of our tRNS signal (Table 1). The induced electrical field strength varied from $\sim 1$ to $1.6,2$, and $3 \mathrm{~V} / \mathrm{m}$ (for intensities of $0.5,0.75,1$, and $1.5 \mathrm{~mA}$, respectively), thus confirming a near linear increase of the induced effect. Note that unlike transcranial direct current stimulation (tDCS), tRNS does not produce tingling or other sensations for the intensities used here.

Applying tRNS to the visual cortex improved detection accuracy, and this effect was significantly larger in the subthreshold stimuli group than in the suprathreshold stimuli group (Fig. $4 A$; significant group main effect tested with a repeated-measures ANOVA, $\left.F_{(1,34)}=7.589, p=0.009\right)$. Plotting detection accuracy as a function of tRNS intensity revealed the typical inverted-Ushaped relationship that was observed for the detection of subthreshold stimuli in individual subjects (Fig. $1 C$, middle, orange) and at the group level (Fig. $4 A$, orange). In contrast, tRNS had very little effect when administered during the detection of suprathreshold stimuli (Figs. $1 C$, middle; $4 A$, blue). The difference between subthreshold and suprathreshold groups was most pronounced for the 0.5 and $1 \mathrm{~mA}$ intensities; however, significance was only reached at uncorrected levels $\left(t_{(18)} \geq 2.357, p \leq 0.030\right.$; preplanned comparisons; corrected $\alpha$ level, 0.0125 ).

The distribution of the individual optimal noise levels (Fig. $4 B$ ) peaked at a tRNS intensity of $1 \mathrm{~mA}$ for the subthreshold stimuli group, whereas it was nearly evenly distributed across all noise conditions in the suprathreshold stimuli group. For the latter group, this suggests that the optimal noise level varied in a 
A

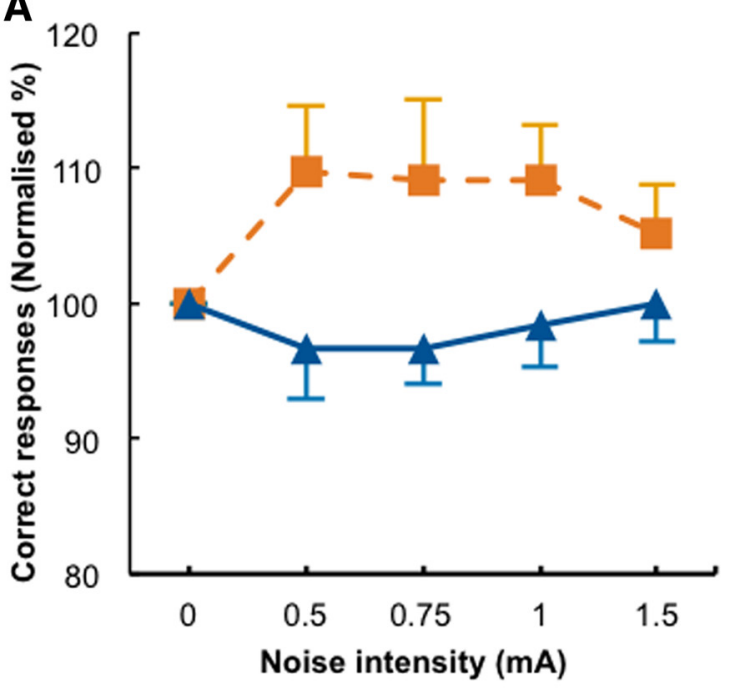

C

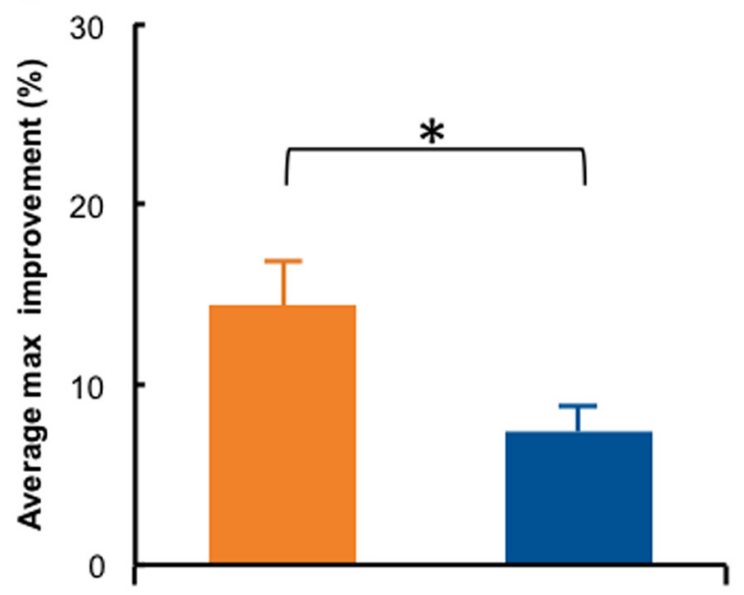

Subthreshold Suprathreshold
B

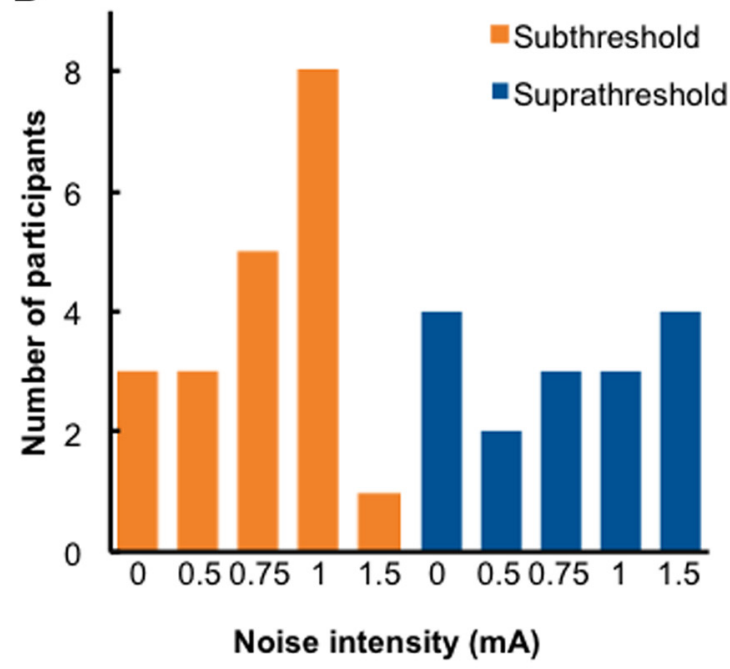

D

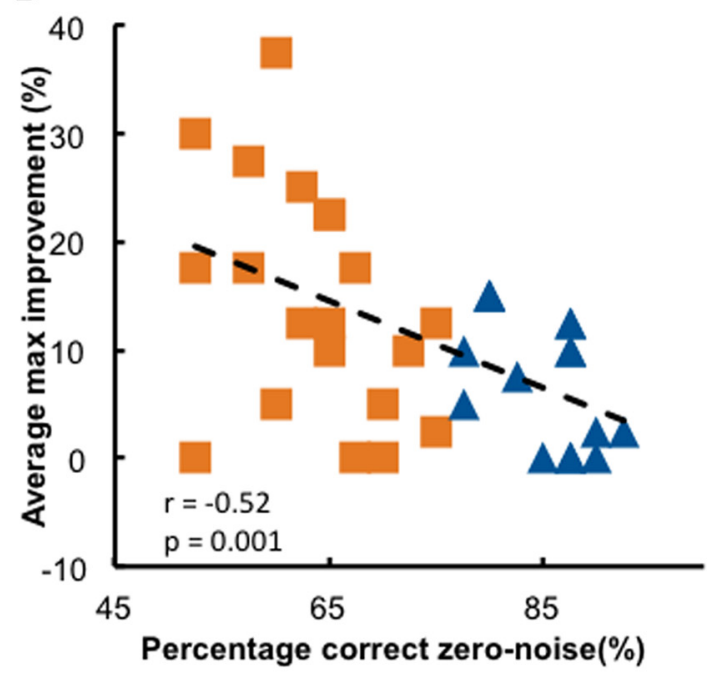

Figure 4. Results when noise is added centrally to the visual cortex (tRNS-noise Experiment). $\boldsymbol{A}$, Detection accuracy relative to the zero noise condition is shown for each noise level in the subthreshold and suprathreshold stimulus groups. $\boldsymbol{B}$, Distribution of the individual optimal noise levels. $\boldsymbol{C}$, The average maximum improvement was significantly different between the subthreshold and suprathreshold groups. ${ }^{*} p<0.05$. D, A significant correlation was present between baseline performance and maximal improvement, indicating that participants with low detection accuracy in the zero noise condition benefited most from adding optimal noise. Conventions are the same as in Figure 3.

close to random manner across subjects. The maximum improvement in accuracy caused by tRNS was significantly greater in the subthreshold stimuli group (13.6\%) compared to the suprathreshold stimuli group $\left(7 \% ; t_{(28.243)}=2.281, p=0.030\right.$, Cohen's $d=0.81$; Fig. $4 C$ ). This was again confirmed by our additional analysis that determined the optimal noise level and the associated detection improvement in an independent manner (subthreshold, 11.1\% accuracy improvement; suprathreshold, $4.8 \%$ accuracy improvement; $t_{(26.457)}=2.397, p=0.024$, Cohen's $d=0.77)$. Similar to the results obtained in the visual-noise experiment, we found a significant correlation $\left(r_{(34)}=-0.52\right.$, $p=0.001)$ between detection accuracy for zero noise trials and the maximum improvement observed for optimal tRNS intensities (Fig. 4D). Two participants in the suprathreshold group reached $100 \%$ accuracy when noise was added with tRNS to the visual cortex. The maximum improvement of these participants (both $12.5 \%$ ) exceeded the group mean of $7.3 \%$. In summary, adding tRNS to the visual cortex induced a pattern of behavioral results highly similar to adding visual noise on the screen, suggesting that the SR phenomenon can be observed when electrical noise is added directly to cortical circuits of human participants engaged in a visual detection task.

\section{Applying tRNS to other cortical areas does not influence visual detection accuracy}

One participant was excluded from the analysis in the subthreshold group because performance in the zero noise condition was below chance level. This left a total number of 19 participants in the analyses ( 9 in the subthreshold group).

Previous research has suggested that the retina and the optical nerve are highly susceptible to alternating currents that can spread to the eyes via the skin, even if the stimulation electrodes are placed far away. Spreading currents that alternate with a frequency of $10-30 \mathrm{~Hz}$ are known to cause phosphenes (Schutter and Hortensius, 2010; Turi et al., 2013), which is a major confound for visual detection accuracy. Even though phosphenes are not evoked by alternating currents with frequencies $>100 \mathrm{~Hz}$ (Turi et al., 2013), as used in our study, we performed a control experiment (tRNS-control) to rule out the possibility that the improvement in detection accuracy was caused by current 

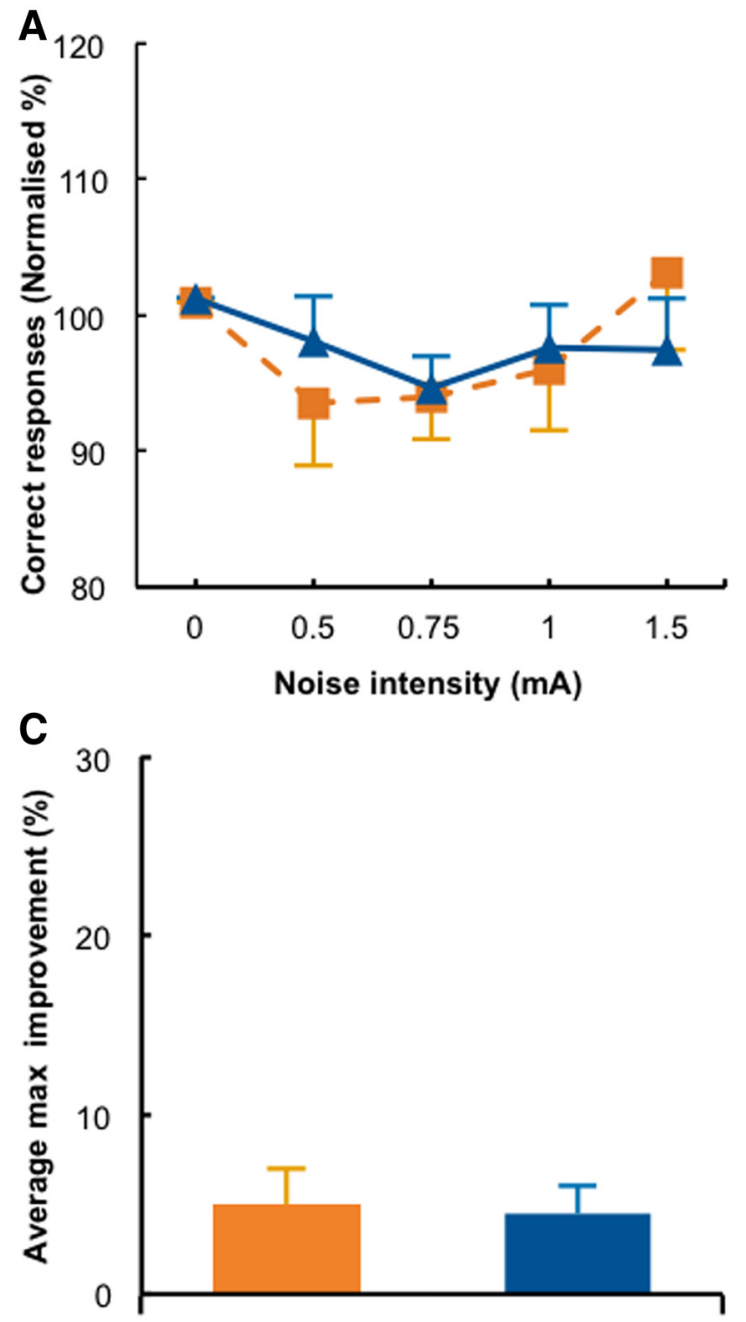

Subthreshold Suprathreshold
B
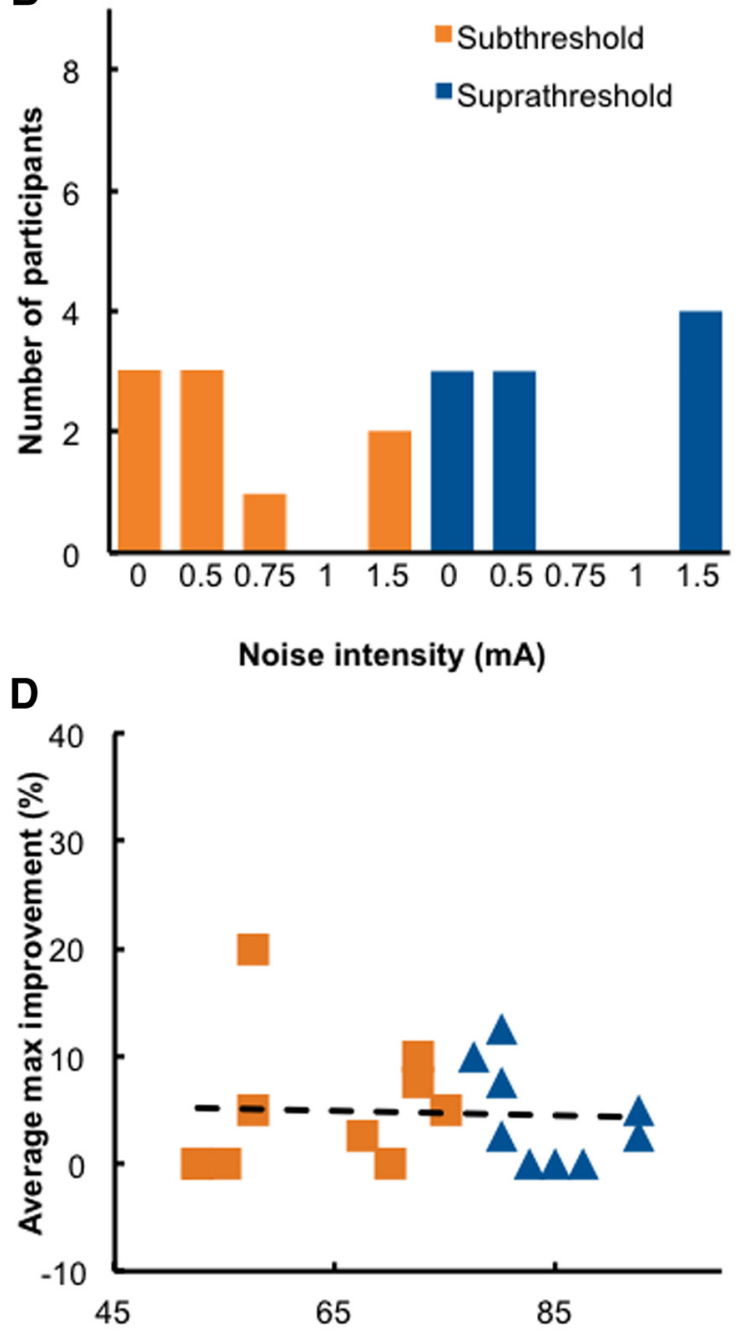

Percentage correct zero-noise(\%)

Figure 5. Results of the tRNS - control experiment. $A$, Detection accuracy relative to the zero noise condition is shown for each noise level in the subthreshold and suprathreshold stimulus groups. B, Distribution of the individual optimal noise levels. $\boldsymbol{C}$, The average maximum improvement was not significantly different between the subthreshold and suprathreshold groups. $\boldsymbol{D}$, The correlation between baseline performance and maximal improvement was not significant. Conventions are the same as in Figure 3.

spreading to the retina and other unspecific tRNS effects. We used the identical protocol and stimulation parameters as in the tRNS-noise experiment, except the electrodes were placed on the vertex and forehead. This setup has been demonstrated to induce more intense phosphenes than the visual cortex-vertex montage used in Experiment 2 (Schutter and Hortensius, 2010).

Applying tRNS over the vertex and forehead did not improve detection accuracy in either group, which exhibited a very similar pattern of results (Figs. $1 C$, third column; $5 \mathrm{~A}$; group main effect and preplanned comparisons, all $p \geq 0.276$ ). In contrast, a significant performance deterioration was found in the suprathreshold group for the $0.75 \mathrm{~mA}$ noise intensity, but only at uncorrected levels $\left(t_{(9)}=-2.685, p=0.025\right.$; corrected $\alpha$ level, 0.0125 ). This null effect is in line with the distribution of individual optimal noise levels (Fig. 5B), which does not show a clear peak. The maximum improvement was small for both groups (subthreshold, 5\%; suprathreshold, 4.4\%; Fig. 5C). In the additional analysis, we did not find a significant difference between subthreshold and suprathreshold groups either $\left(t_{(17)}=0.533\right.$, $p=0.601$ ), and effect sizes were generally small (Table 2 ). The correlation between detection accuracy for zero noise trials and the maximum improvement observed for optimal tRNS intensities did not reach significance, indicating that the effects caused by tRNS to the frontal lobe did not depend on detection accuracy in the zero noise condition (Fig. 5D). Finally, there was a significant difference between the maximum improvement in the subthreshold groups of the tRNS-control experiment and tRNSnoise experiment $\left(t_{(26)}=5.447, p=0.028\right)$, indicating that the addition of noise to the visual cortex significantly enhanced detection performance when compared to our control experiment.

\section{Discussion}

This study provides direct evidence for enhanced detection of subthreshold visual stimuli when tRNS is added centrally to the visual cortex at an optimal intensity. When tRNS intensities were increased (ranging from 0 to $1.5 \mathrm{~mA}$ ), the detection accuracy of subthreshold visual stimuli changed according to an inverted-Ushaped function, typical of the SR phenomenon. When the optimal level of noise was added to the visual cortex, detection performance improved significantly relative to the zero noise 
condition. It also improved to a similar extent when noise was added to the visual stimuli. The noise-enhancing effect was not observed for suprathreshold stimuli.

Optimal noise intensities varied substantially across individuals, a finding that is well known when noise is added to the stimulus directly (Collins et al., 1996; Martínez et al., 2007). We accommodated this variability by determining the optimal noise intensities at the individual level rather than at group level. A split-half analysis was used to minimizes the risk of statistical "double dipping." This analysis confirmed that both optimal visual noise and optimal tRNS noise had a signal-enhancing effect for subthreshold but not suprathreshold stimuli, an important characteristic of the SR phenomenon. Additionally, when intensities were weaker or stronger than optimal, there was little to no effect on detection accuracy. When plotted, these results show an inverted-U-shaped tuning curve, a hallmark feature of the SR phenomenon (Moss et al., 2004). It is worth noting that SR effects induced with tRNS were qualitatively similar to those observed when noise was added to the visual stimulus, even though the effect magnitude was somewhat larger in the visual-noise than the tRNS-noise experiment. Thus, our results suggest that online tRNS adds noise to neural processing that has a similar effect as adding noise to the visual stimulus.

To determine whether tRNS induced electrical noise in the cortex, we used simulations based on a spherical head model. Simulation results suggested that the induced electrical field ranges from $\sim 1$ to $3 \mathrm{~V} / \mathrm{m}$ (scaling quasi-linearly with tRNS intensity), and that all stimulation frequencies were transmitted to the cortex. Note that the estimated field strength is too small to directly depolarize cortical neurons or to cause conscious sensations (e.g., tingling of the skin) in the participants; however, it is sufficiently strong to modulate cortical processing after continuous application (Terney et al., 2008; Fertonani et al., 2011). It is known that the effect of transcranial current stimulation (tCS) also depends on the morphology of the stimulated neuronal tissue (Radman et al., 2009). Because of the complex morphology of the visual cortex, we cannot exclude the possibility that different retinotopic coordinates received different levels of stimulation. In the design of our experiment, we attempted to reduce the potential for bias by presenting the stimulus five times at each of the eight locations in each experimental run. Moreover, a post hoc control analysis testing whether the tRNS effect differed for targets in the left versus right and lower versus upper visual hemifields did not reveal systematic modulation.

The effects we observed are likely to reflect the acute/online influence of tRNS rather than aftereffects that accumulate over time, since stimulation was only applied for short intervals (2.04 s) and followed by long periods of no stimulation. Furthermore, Pirulli et al. (2013) reported that tRNS only influenced behavioral performance when applied during (online) a perceptual learning task, and not when applied before task learning (off-line). They hypothesized that tRNS could induce temporal summation of small depolarizing currents that interact with the ongoing activity of cortical neurons. The order of tRNS blocks was also randomized over participants to prevent any order or intensity dependent aftereffects. We also eliminated the possibility that results may be due to a general increase in arousal, tRNS-induced phosphenes, or other unspecific effects through our tRNS-control experiment. Detection accuracy did not improve when a control electrode montage was used. The sample size in the tRNS-control experiment was smaller than the sample size in the tRNSnoise experiment. However, a power analysis considering the experimentally determined effect size of 0.26 for the tRNS-control experiment and a power of 0.6 (Table 2, power of the control analysis of the tRNS-noise experiment) revealed that 344 participants would be needed to reach a significant effect for $\alpha=0.05$. This suggests that the lack of a significant effect in the tRNScontrol experiment does not primarily result from the smaller sample size, but rather from the low efficacy of the control stimulation.

Our study provides behavioral evidence for an SR mechanism when tRNS is added to neural networks in human cortex. This suggests that not only single neurons, but also cortical networks processing visual stimuli, exhibit nonlinear system properties, extending pervious work showing SR effects at the neural network level in vitro (Swain and Longtin, 2006; Manjarrez et al., 2007). Our findings are also consistent with previous studies that demonstrate SR based detection enhancement even though the to-be-detected stimulus and the noise were presented to different modalities or effectors (Kitajo et al., 2003; Manjarrez et al., 2007; Lugo et al., 2008). Note, however, that when both noise and stimuli are presented to a receptor, one cannot exclude that SR effects emerge subcortically, e.g., in the superior colliculus, a well-known multisensory integration area. Given what is known about the spread of noninvasive brain stimulation within cortex, it is highly unlikely that tRNS currents were sufficiently strong to modulate subcortical structures. Therefore, our results suggest that neuronal populations in cortex can benefit from the direct application of noise.

Our finding also lends indirect support to the hypothesis that noninvasive brain stimulation techniques in general might modulate brain function via an SR mechanism, but that the effect depends on the level of noise and brain activity (Miniussi et al., 2013). This proposal was first tested by applying transcranial magnetic stimulation (TMS) pulses with an intensity determined via the phosphene threshold to visual cortex and measuring its influence on detection accuracy. However, findings differed across studies and could not be unequivocally interpreted, partly because it is not clear whether the TMS pulses added noise to neural processing (Schwarzkopf et al., 2011) or suppressed task-related activity (Harris et al., 2008). Others have shown that also cathodal tDCS can enhance the perception of difficult to detect stimuli (i.e., obliquely oriented gratings of higher spatial frequencies) when compared to easier to detect stimuli (Richard et al., 2015). The authors suggested that tDCS exerted its influence on visual perception by modulating the signal-to-noise ratio of neurons in visual cortex and speculated that this effect has been mediated by an SR mechanism. Even though our data cannot directly speak to TMS or tDCS experiments, our results are in support of the idea that neural processing at the network level exhibits nonlinear system properties that are sensitive to the SR phenomenon, which can be quantified via behavioral readouts (Fertonani and Miniussi, 2016). A major advantage of tRNS is that the signal has well-defined noise characteristics and can be applied continuously during the task without depolarizing neurons.

\section{Interpretational issues}

One limitation of our study is that despite the clear behavioral evidence, we did not directly measure neural signals and how they might have changed by adding tRNS to the scalp. Based on previous studies that used an identical electrode placement and our modeling, we hypothesized that the induced currents reached mainly visual cortex (Neuling et al., 2012). However, drawing firm conclusions on which neural areas or processes have been influenced will require the use of neuroimaging methods (preferably electroencephalography) during tRNS application. 


\section{Conclusion}

In summary, our results highlight the potential to exploit SR mechanisms via tRNS to modulate human behavior via noninvasive brain stimulation. Even though we used visual perception as a model system, tRNS can be applied to all cortical areas, indicating that SR effects can be used for all functions that depend on nonlinear cortical dynamics.

\section{References}

Brainard DH (1997) The psychophysics toolbox. Spat Vis 10:433-436. CrossRef Medline

Bromm B (1968) Die natrium-gleichrichtung der unterschwellig erregten membran in der quantitativen formulierung der ionentheorie. Pflügers Archiv 302:233-244. Medline

Cameron EL, Tai JC, Carrasco M (2002) Covert attention affects the psychometric function of contrast sensitivity. Vision Res 42:949-967. CrossRef Medline

Chaieb L, Antal A, Paulus W (2015) Transcranial random noise stimulation-induced plasticity is NMDA-receptor independent but sodium-channel blocker and benzodiazepines sensitive. Frontiers Neurosci 9:125. CrossRef

Collins JJ, Chow CC, Imhoff TT (1995) Stochastic resonance without tuning. Nature 376:236-238. CrossRef Medline

Collins JJ, Imhoff TT, Grigg P (1996) Noise-enhanced tactile sensation. Nature 383:770. CrossRef Medline

Faul F, Erdfelder E, Lang AG, Buchner A (2007) G*Power 3: a flexible statistical power analysis program for the social, behavioral, and biomedical sciences. Behav Res Methods 39:175-191. CrossRef Medline

Fertonani A, Miniussi C (2016) Transcranial electrical stimulation: what we know and do not know about mechanisms. Neuroscientist. 1:15. CrossRef Medline

Fertonani A, Pirulli C, Miniussi C (2011) Random noise stimulation improves neuroplasticity in perceptual learning. J Neurosci 31:1541615423. CrossRef Medline

Fertonani A, Ferrari C, Miniussi C (2015) What do you feel if I apply transcranial electric stimulation? Safety, sensations and secondary induced effects. Clin Neurophysiol 126:2181-2188. CrossRef Medline

Gingl Z, Kiss L, Moss F (1995) Non-dynamical stochastic resonance: Theory and experiments with white and arbitrarily coloured noise. Europhys Lett 29:191. CrossRef

Harris JA, Clifford CW, Miniussi C (2008) The functional effect of transcranial magnetic stimulation: signal suppression or neural noise generation? J Cogn Neurosci 20:734-740. CrossRef Medline

Kitajo K, Nozaki D, Ward LM, Yamamoto Y (2003) Behavioral stochastic resonance within the human brain. Phys Rev Lett 90:218103. CrossRef Medline

Kleiner M, Brainard D, Pelli D (2007) What's new in Psychtoolbox-3? Perception 36:ECVP Abstract Supplement.

Lugo E, Doti R, Faubert J (2008) Ubiquitous crossmodal Stochastic Resonance in humans: auditory noise facilitates tactile, visual and proprioceptive sensations. PLoS One 3:e2860. CrossRef Medline

Manjarrez E, Mendez I, Martinez L, Flores A, Mirasso CR (2007) Effects of auditory noise on the psychophysical detection of visual signals: crossmodal stochastic resonance. Neurosci Lett 415:231-236. CrossRef Medline

Martínez L, Pérez T, Mirasso CR, Manjarrez E (2007) Stochastic resonance in the motor system: effects of noise on the monosynaptic reflex pathway of the cat spinal cord. J Neurophysiol 97:4007-4016. CrossRef Medline

Miniussi C, Harris JA, Ruzzoli M (2013) Modelling non-invasive brain stimulation in cognitive neuroscience. Neurosci Biobehav Rev 37: 1702-1712. CrossRef Medline

Moss F, Ward LM, Sannita WG (2004) Stochastic resonance and sensory information processing: a tutorial and review of application. Clin Neurophysiol 115:267-281. CrossRef Medline

Neuling T, Wagner S, Wolters CH, Zaehle T, Herrmann CS (2012) Finiteelement model predicts current density distribution for clinical applications of tDCS and tACS. Front Psychiatry 3:83. Medline

Pelli DG (1997) The VideoToolbox software for visual psychophysics: transforming numbers into movies. Spat Vis 10:437-442. CrossRef Medline

Pirulli C, Fertonani A, Miniussi C (2013) The role of timing in the induction of neuromodulation in perceptual learning by transcranial electric stimulation. Brain Stim 6:683-689. CrossRef Medline

Radman T, Ramos RL, Brumberg JC, Bikson M (2009) Role of cortical cell type and morphology in subthreshold and suprathreshold uniform electric field stimulation in vitro. Brain Stimul 2:215-228. CrossRef Medline

Riani M, Simonotto E (1994) Stochastic resonance in the perceptual interpretation of ambiguous figures - a neural-network model. Phys Rev Lett 72:3120-3123. CrossRef Medline

Richard B, Johnson AP, Thompson B, Hansen BC (2015) The effects of tDCS across the spatial frequencies and orientations that comprise the contrast sensitivity function. Front Psychiatry. 6:1784. CrossRef Medline

Sasaki H, Sakane S, Ishida T, Todorokihara M, Kitamura T, Aoki R (2008) Subthreshold noise facilitates the detection and discrimination of visual signals. Neurosci Lett 436:255-258. CrossRef Medline

Schoen I, Fromherz P (2008) Extracellular stimulation of mammalian neurons through repetitive activation of $\mathrm{Na}+$ channels by weak capacitive currents on a silicon chip. J Neurophysiol 100:346-357. CrossRef Medline

Schutter DJ, Hortensius R (2010) Retinal origin of phosphenes to transcranial alternating current stimulation. Clin Neurophysiol 121:1080-1084. CrossRef Medline

Schwarzkopf DS, Silvanto J, Rees G (2011) Stochastic resonance effects reveal the neural mechanisms of transcranial magnetic stimulation. J Neurosci 31:3143-3147. CrossRef Medline

Simonotto E, Riani M, Seife C, Roberts M, Twitty J, Moss F (1997) Visual perception of stochastic resonance. Phys Rev Lett 78:1186-1189. CrossRef

Swain PS, Longtin A (2006) Noise in genetic and neural networks. Chaos 16:026101. CrossRef Medline

Terney D, Chaieb L, Moliadze V, Antal A, Paulus W (2008) Increasing human brain excitability by transcranial high-frequency random noise stimulation. J Neurosci 28:14147-14155. CrossRef Medline

Tong F, Meng M, Blake R (2006) Neural bases of binocular rivalry. Trends Cogn Sci 10:502-511. CrossRef Medline

Truong DQ, Hüber M, Xie X, Datta A, Rahman A, Parra LC, Dmochowski JP, Bikson M (2014) Clinician accessible tools for GUI computational models of transcranial electrical stimulation: BONSAI and SPHERES. Brain Stimul 7:521-524. CrossRef Medline

Turi Z, Ambrus GG, Janacsek K, Emmert K, Hahn L, Paulus W, Antal A (2013) Both the cutaneous sensation and phosphene perception are modulated in a frequency-specific manner during transcranial alternating current stimulation. Restor Neurol Neurosci 31:275-285. CrossRef

Ward LM, Desai S, Rootman D, Tata M, Moss F (2001) Noise can help as well as hinder seeing and hearing. Bull Am Phys Soc 46:N23.002.

Watson AB, Pelli DG (1983) QUEST: A Bayesian adaptive psychometric method. Atten Percept Psycho 33:113-120. CrossRef Medline

Wunderlich K, Schneider KA, Kastner S (2005) Neural correlates of binocular rivalry in the human lateral geniculate nucleus. Nat Neurosci 8: 1595-1602. CrossRef Medline

Zeng FG, Fu QJ, Morse R (2000) Human hearing enhanced by noise. Brain Res 869:251-255. CrossRef Medline 